\title{
Energy dependence of hadron spectra and multiplicities in $p+p$ interactions
}

\section{Szymon Puławski for the NA61/SHINE Collaboration}

Institute of Physics, University of Silesia

E-mail: s.pulawski@cern.ch

The NA61/SHINE experiment at the CERN SPS aims to discover the critical point of strongly interacting matter and study the properties of the onset of deconfinement. In order to reach these goals measurements of hadron production properties are performed in nucleus-nucleus, protonproton and proton-nucleus interactions as a function of collision energy and size of the colliding nuclei.

Inclusive spectra of identified hadrons in $p+p$ interactions at the SPS energies are presented as a function of transverse momentum, transverse mass and rapidity. The results are compared with the world data and theoretical models.

9th International Workshop on Critical Point and Onset of Deconfinement - CPOD2014,

17-21 November 2014

ZiF (Center of Interdisciplinary Research), University of Bielefeld, Germany

* Speaker. 


\section{Experimental data and analysis methods}

This paper presents inclusive spectra of identified hadrons produced in inelastic $p+p$ interactions at 20,31, 40, 80, $158 \mathrm{GeV} / \mathrm{c}$. Three analysis methods were used:

- For $\pi^{-}$spectra the $h^{-}$method [1], which is based on the fact that the majority of negatively charged particles are $\pi^{-}$mesons. The contribution of other particles is subtracted using calculations based on the EPOS model [2],

- for $\pi^{+}, \pi^{-}, K^{+}, K^{-}$and $p, \bar{p}$ the $d E / d x$ method, which uses information on particle energy loss $(d E / d x)$ in the TPC gas to identify particles,

- for $\pi^{+}, \pi^{-}, K^{+}, K^{-}$and $p, \bar{p}$ the $t o f-d E / d x$ method, which in addition to the $d E / d x$ information uses time of flight (tof) measurements. Combined tof $-d E / d x$ analysis provides excellent separation of different hadron species close to the mid-rapidity region.

Results were corrected for trigger and event selection biases, contribution of non-target interactions as well as detector inefficiencies and feed-down from weak decays and secondary interactions.

\section{Results on single-particle spectra}

Spectra of transverse mass $m_{T}$ of negatively and positively charged pions, kaons, protons and $\Lambda$ hyperons produced in inelastic $\mathrm{p}+\mathrm{p}$ interactions at $158 \mathrm{GeV} / \mathrm{c}$ at mid-rapidity are presented in Fig. 1 (left). The corresponding data for central $\mathrm{Pb}+\mathrm{Pb}$ collisions measured by $\mathrm{NA} 49[3,4,6]$ are shown in the right panel of Fig. 1 (right). These data sets were fitted using the simplified blast wave model parameterization [7]: $\frac{d N_{i}}{m_{T} d m_{T} d y}=A_{i} m_{T} K_{1}\left(\frac{m_{T} \cosh \rho}{T}\right) I_{0}\left(\frac{p_{T} \sinh \rho}{T}\right)$. The transverse flow velocity $\beta_{T}$ was calculated from the equation $\rho=\tanh ^{-1} \beta_{T}$.

The value of $\beta_{T}$ is much higher in central $\mathrm{Pb}+\mathrm{Pb}$ than in $\mathrm{p}+\mathrm{p}$ reactions. Transverse mass spectra are approximately exponential in $\mathrm{p}+\mathrm{p}$ interactions. The exponential dependence is modified by the transverse flow in central $(7 \%) \mathrm{Pb}+\mathrm{Pb}$ collisions. Transverse collective flow in $\mathrm{Pb}+\mathrm{Pb}$ can explain the significant spectra shape difference between $\mathrm{p}+\mathrm{p}$ and central $\mathrm{Pb}+\mathrm{Pb}$ reactions.

Rapidity distributions of $\pi^{-}$produced in inelastic $p+p$ collisions [1] and energy dependence of the width $\sigma$ of the rapidity distribution divided by beam rapidity $\left(y_{\text {beam }}\right)$ or by the hydrodynamic model prediction $\left(\sigma_{L S}\right)[8,9]$ are presented in Fig. 2. The shape of the rapidity distribution is approximately Gaussian, however the best fit was obtained by a sum of two Gauss distributions. The width of the $\pi^{-}$rapidity distribution divided by beam rapidity decreases with the collision energy. $\sigma / \sigma_{L S}$ and $\sigma / y_{\text {beam }}$ are smaller in $\mathrm{p}+\mathrm{p}$ than in $\mathrm{Pb}+\mathrm{Pb}$ interactions. No significant difference of the energy dependence is observed for the width of the $\pi^{-}$rapidity distribution in $\mathrm{p}+\mathrm{p}$ and $\mathrm{Pb}+\mathrm{Pb}$ interactions.

The results presented in this paper are an important step towards the study of the system size dependence of the signals of the onset of deconfinement observed in central $\mathrm{Pb}+\mathrm{Pb}$ collisions, the "kink", "horn" and "step" $[4,5]$. The $\pi$ multiplicity at SPS energies shown in Fig. 3 increases faster in central $\mathrm{Pb}+\mathrm{Pb}$ than in inelastic $\mathrm{p}+\mathrm{p}$ collisions (kink) and the two dependencies cross each other at about $40 \mathrm{~A} \mathrm{GeV/c}$. 

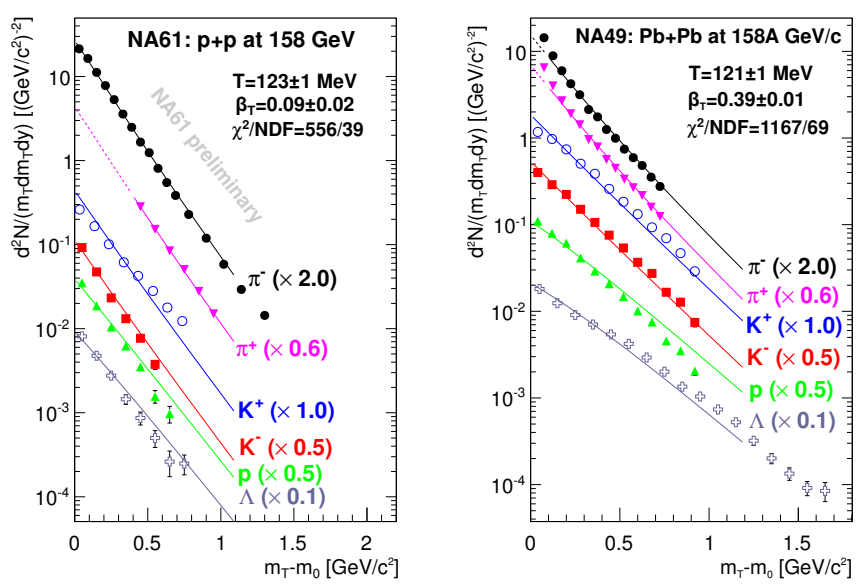

Figure 1: Transverse mass spectra at mid-rapidity measured by the NA61/SHINE experiment in inelastic $\mathrm{p}+\mathrm{p}$ interactions (left) and corresponding results of NA49 results from central $\mathrm{Pb}+\mathrm{Pb}$ collisions at $158 \mathrm{~A} \mathrm{GeV}$ (right).
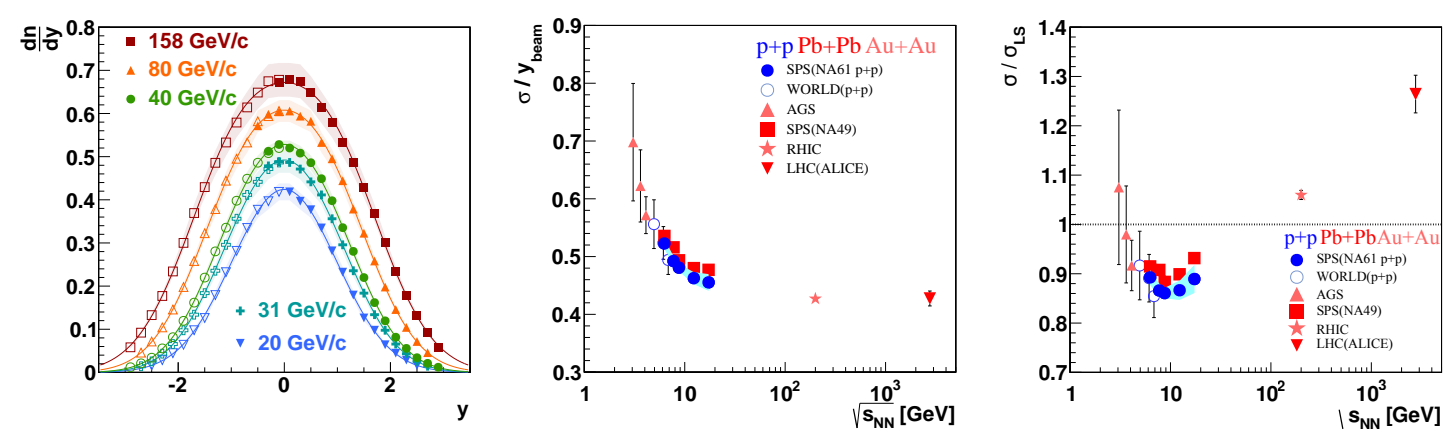

Figure 2: $\pi^{-}$rapidity distributions measured in inelastic $\mathrm{p}+\mathrm{p}$ collisions at 20, 31, 40, 80 and $158 \mathrm{GeV} / \mathrm{c}$ (left). Dependence of the scaled width of the rapidity distribution on collision energy (center and right). World data are taken from Refs. [10,11, 12]. Results for the width of rapidity distributions are not corrected for isospin effects.

Preliminary NA61/SHINE measurements allow to significantly improve the world data on the inverse slope parameter $T$ of transverse mass spectra of kaons [14] as well as the ratio of $K^{+}$and $\pi^{+}$yields at mid-rapidity $[15,16]$.

The $\mathrm{m}_{T}$ spectra were parametrized by the exponential function:

$$
\frac{d^{2} n}{d p_{T} d y}=\frac{S p_{T}}{T^{2}+m_{K} T} \exp \left(-\frac{\sqrt{p_{T}^{2}+m_{K}^{2}}-m_{K}}{T}\right),
$$

where $S$ and $T$ are the yield integral and the inverse slope parameter, respectively. The $S$ and $T$ parameters were fitted to spectra of $K^{+}$and $K^{-}$mesons at mid-rapidity as shown in Fig. 4. 

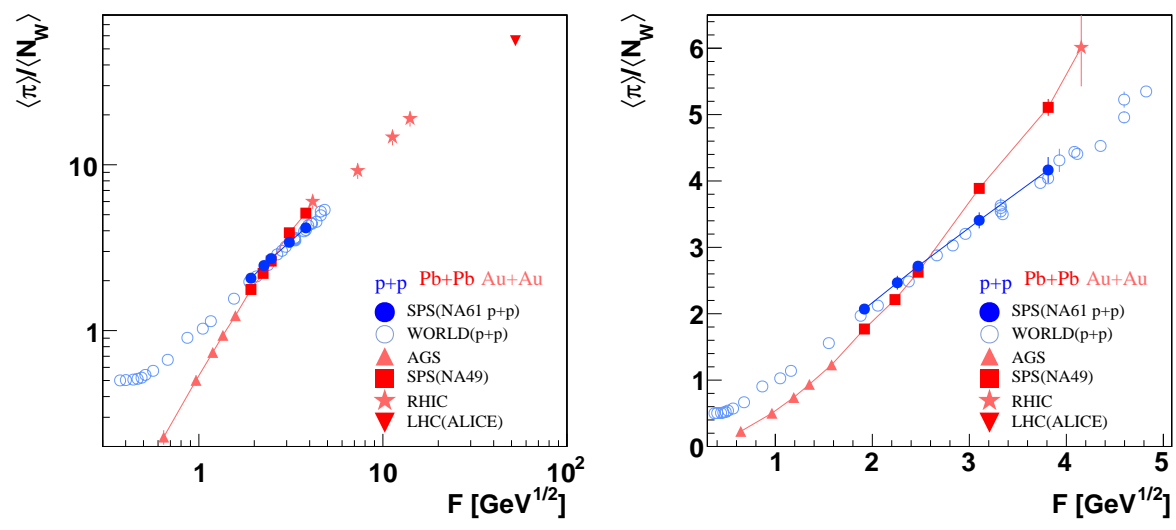

Figure 3: Energy dependence of $\pi$ multiplicity as a function of collision energy. ALICE points were derived from Refs. [11, 13].
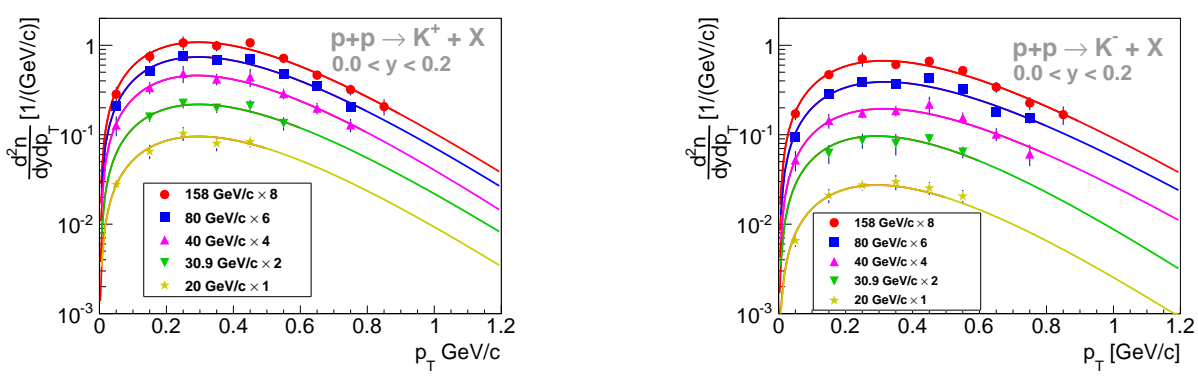

Figure 4: Transverse momentum spectra of $K^{+}$and $K^{-}$mesons produced at $y \approx 0$ in inelastic p+p interactions measured by NA61/SHINE.

Figure 5 presents the energy dependence of the inverse slope parameter $T$ of kaons. Surprisingly the NA61/SHINE results from inelastic p+p collisions exhibit rapid changes like observed in central $\mathrm{Pb}+\mathrm{Pb}$ interactions. World data for $\mathrm{p}+\mathrm{p}$ and $\mathrm{Pb}+\mathrm{Pb} / \mathrm{Au}+\mathrm{Au}$ reactions are plotted for comparison and were taken from Refs. [14, 17, 18, 19].

The acceptance of the identification methods used at the moment by NA61/SHINE does not allow to measure spectra of positive pions close to mid-rapidity. Negatively charged pion spectra were measured by NA61/SHINE [1] using the $h^{-}$method. This method cannot be used for positively charged pions due to the large contribution of protons and positive kaons.

Therefore, in order to determine the mid-rapidity yield of positively charged pions the following procedure was used:

- The ratio of measured $\pi^{+}$and $\pi^{-}$yields was calculated (within the acceptance of the tof$d E / d x$ and $d E / d x$ methods) and compared with model predictions as a function of collision energy, see Fig. 6. The agreement between the data and the EPOS model [2] predictions is better than $0.1 \%$. 

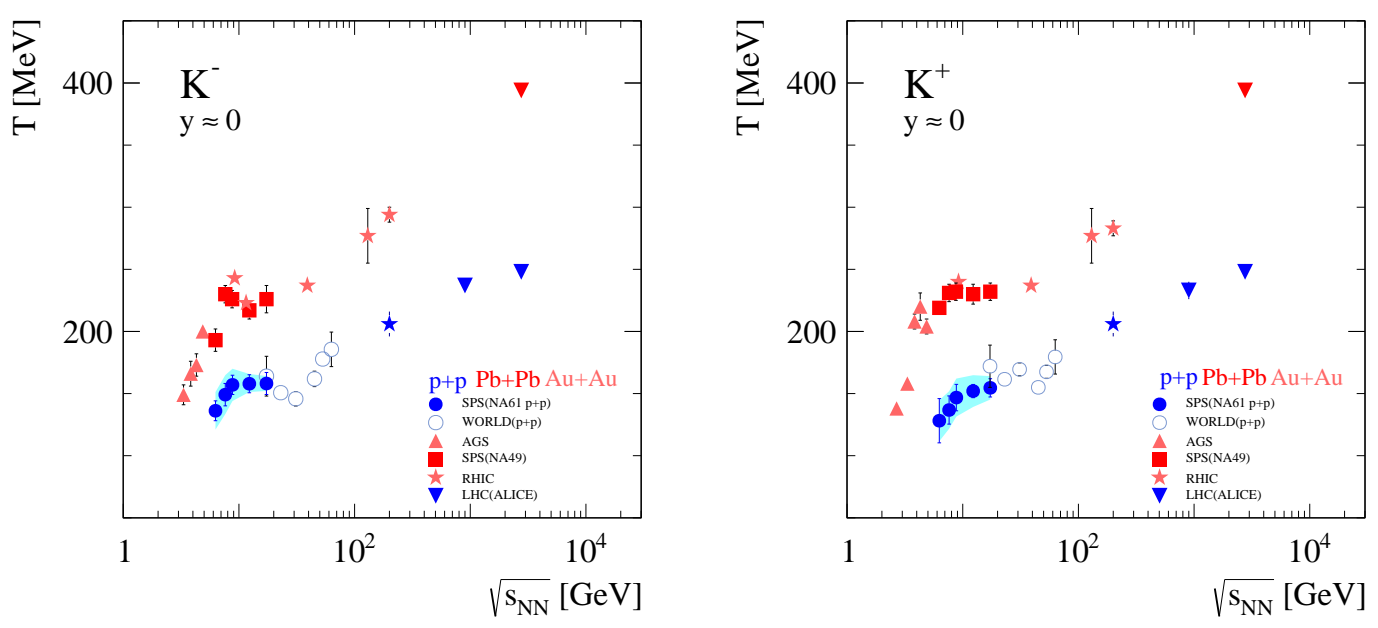

Figure 5: Energy dependence of the inverse slope parameter $T$ of transverse mass spectra of kaons for inelastic $\mathrm{p}+\mathrm{p}$ interactions and central $\mathrm{Pb}+\mathrm{Pb} / \mathrm{Au}+\mathrm{Au}$ collisions.

- The mid-rapidity ratio of the $\pi^{+}$and $\pi^{-}$yields was calculated from the EPOS model.

- The $\pi^{+}$mid-rapidity yield was calculated as the product of the measured $\pi^{-}$yield at $y=0$, the measured $\frac{\pi+}{\pi^{-}}$ratio in the $t o f-d E / d x$ acceptance and the EPOS correction factor:

$$
\pi^{+}(y=0)=\pi^{-}(y=0) \frac{\pi^{+}}{\pi^{-}}(t o f-d E / d x) C_{M C}
$$

where:

$$
C_{M C}=\left[\frac{\frac{\pi^{+}}{\pi^{-}}(y=0)}{\frac{\pi^{+}}{\pi^{-}}(\text {tof })}\right]_{M C} \approx 5 \% .
$$

The final correction factor as a function of energy is shown in Fig. 6.

The energy dependence of the $K^{+} / \pi^{+}$and $K^{-} / \pi^{-}$ratios at mid-rapidity for inelastic p+p interactions and central $\mathrm{Pb}+\mathrm{Pb} / \mathrm{Au}+\mathrm{Au}$ collisions is presented in Fig. 7. Surprisingly the NA61/SHINE data suggest that even in inelastic $\mathrm{p}+\mathrm{p}$ interactions the energy dependence of the $K^{+} / \pi^{+}$ratio exhibits rapid changes in the SPS energy range. However, the horn structure is significantly reduced/modified in comparison to that observed in central $\mathrm{Pb}+\mathrm{Pb}$ collisions. Additionally world data $[15,16,20,19,13]$ is plotted to establish the trend outside the SPS energy range. The NA61/SHINE results were compared to theoretical models in Ref. [25], namely EPOS [2], UrQMD [22, 21], Pythia 8 [24] and HSD [23]. Figure 8 shows that these models do not describe well the NA61/SHINE results on $\mathrm{p}+\mathrm{p}$ interactions. The high precision of the measurements should allow for significant improvement of the models.

Proton transverse momentum spectra and mean transverse mass $\left\langle m_{T}\right\rangle$ around mid-rapidity in inelastic $p+p$ interactions at SPS energies are presented and compared with theoretical models in 

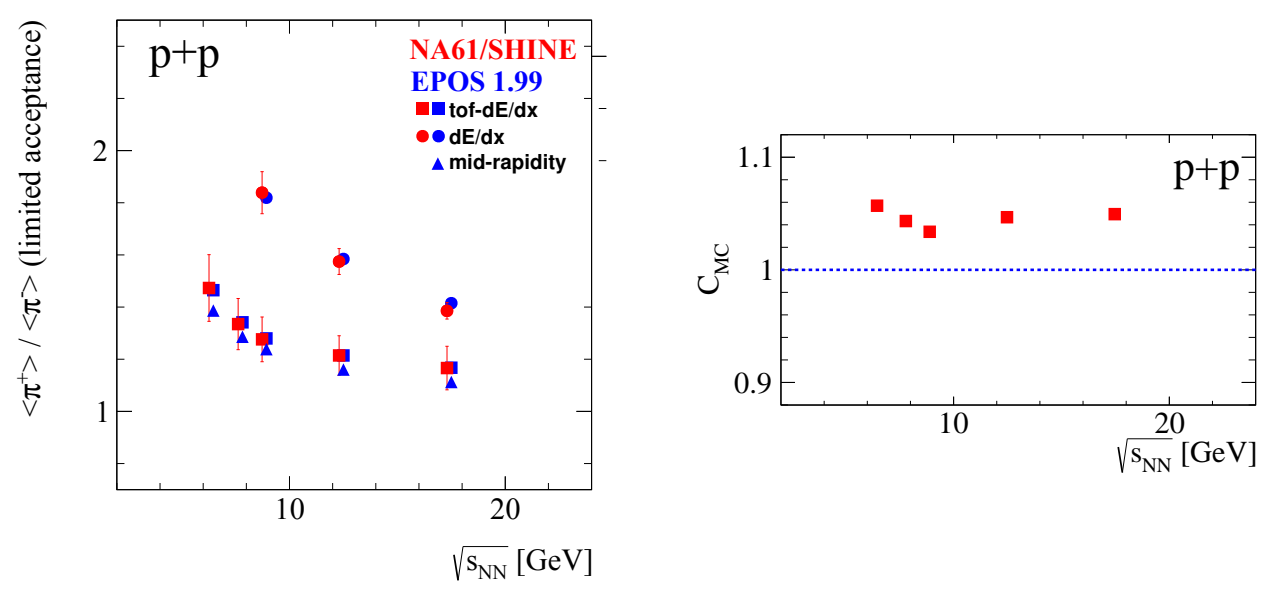

Figure 6: The ratio of $\pi^{+}$and $\pi^{-}$yields in inelastic $p+p$ interactions as a function of collision energy. The ratio was calculated in the acceptance of the tof $-d E / d x$ and $d E / d x$ identification methods. The NA61/SHINE results are compared to predictions of the EPOS model (left). Correction factor used to obtain $\pi^{+}$yields from the measured $\pi^{-}$yields (right), see Eq. 2.3.
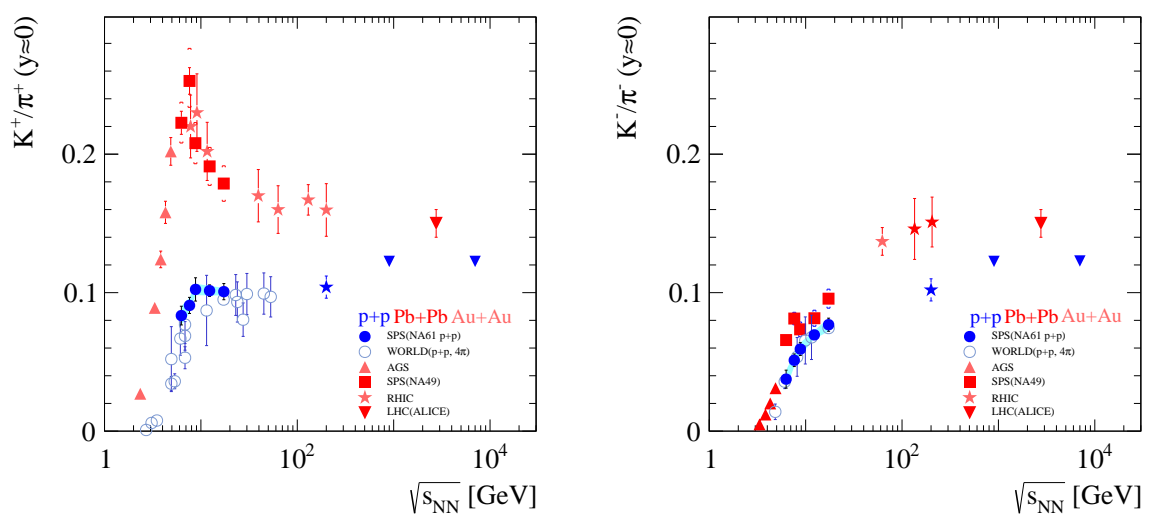

Figure 7: The $K^{+} / \pi^{+}$and $K^{-} / \pi^{-}$ratios in inelastic $\mathrm{p}+\mathrm{p}$ and central $\mathrm{Pb}+\mathrm{Pb} / \mathrm{Au}+\mathrm{Au}$ reactions plotted versus collision energy.

Fig. 9. Mean $m_{T}$ of protons was calculated from the data parametrization:

$$
\frac{d^{2} n}{d p_{T} d y}=\frac{S p_{T}}{T^{2}+m_{p} T} \exp \left(-\frac{\sqrt{p_{T}^{2}+m_{p}^{2}}-m_{p}}{T}\right) .
$$

It increases slowly with collision energy. Neither UrQMD nor HSD describe this behavior [25].

\section{Conclusions}

The NA61/SHINE measurements show that $p+p$ collisions are unexpectedly interesting. Surprisingly the NA61/SHINE results suggest that even in inelastic $p+p$ interactions the energy depen- 

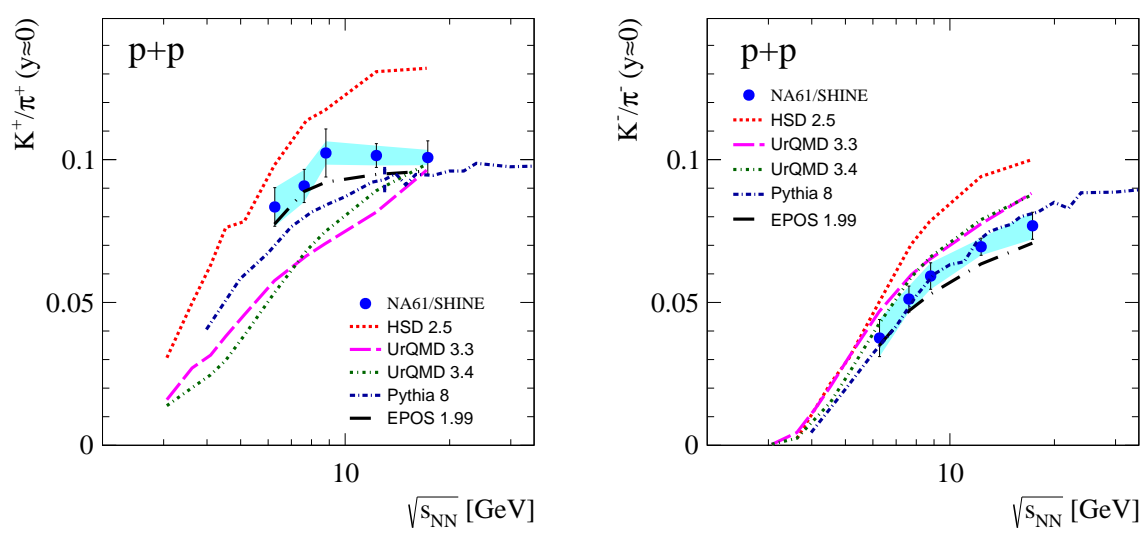

Figure 8: Comparison of $K / \pi$ ratios in inelastic $\mathrm{p}+\mathrm{p}$ collisions at SPS energies with theoretical models.
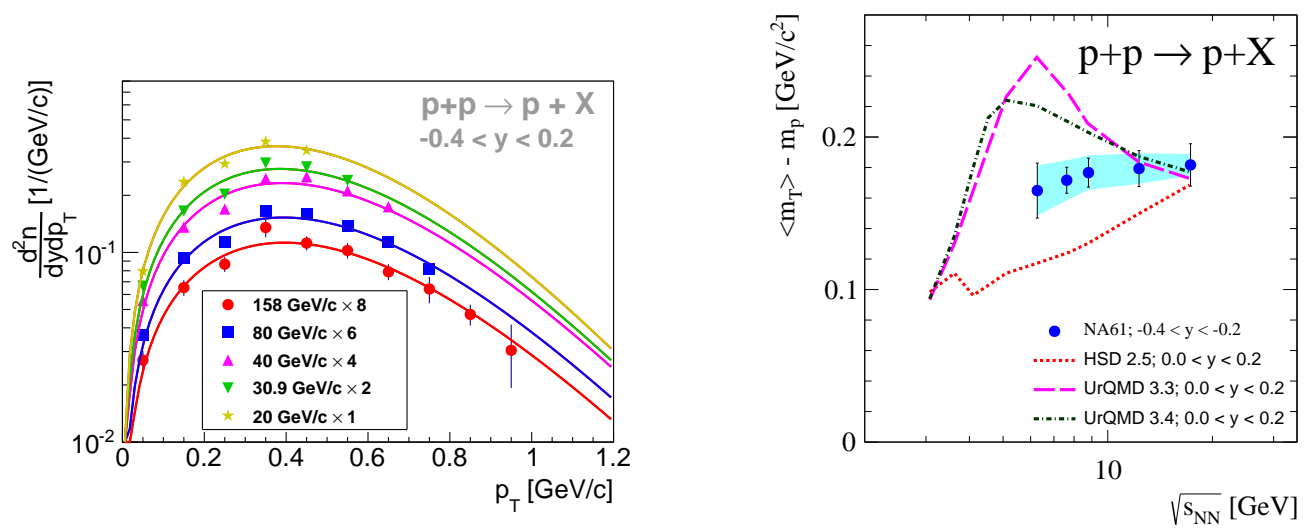

Figure 9: Proton transverse momentum spectra (left) and mean transverse mass $m_{T}$ (right) around midrapidity in inelastic $\mathrm{p}+\mathrm{p}$ interactions at SPS energies compared with theoretical models.

dence of the $K^{+} / \pi^{+}$ratio and the inverse slope parameter of kaon transverse mass spectra exhibits rapid changes in the SPS energy range. However, the structures, e.g. the step and horn, are significantly reduced/modified compared to those observed in central $\mathrm{Pb}+\mathrm{Pb}$ collisions. High precision NA61/SHINE data present challenges for models and should allow their improvement.

\section{Acknowledgments}

This work was supported by the National Science Center of Poland (grant 2014/12/T/ST2/00692, grant 2013/11/N/ST2/03879, grant 2012/04/M/ST2/00816).

\section{References}

[1] N. Abgrall et al. Measurement of negatively charged pion spectra in inelastic $\mathrm{p}+\mathrm{p}$ interactions at $p_{l a b}=20,31,40,80$ and $158 \mathrm{GeV} /$ c. Eur.Phys.J., C74(3):2794, 2014. 
[2] T. Pierog and K. Werner. EPOS Model and Ultra High Energy Cosmic Rays. Nucl.Phys.Proc.Suppl., 196:102-105, 2009.

[3] C. Alt et al. Energy and centrality dependence of anti-p and p production and the anti-Lambda/anti-p ratio in $\mathrm{Pb}+\mathrm{Pb}$ collisions between 20/A-GeV and 158/A-Gev. Phys.Rev., C73:044910, 2006.

[4] C. Alt et al. Pion and kaon production in central $\mathrm{Pb}+\mathrm{Pb}$ collisions at 20-A and 30-A-GeV: Evidence for the onset of deconfinement. Phys.Rev., C77:024903, 2008.

[5] Marek Gazdzicki, Mark Gorenstein, and Peter Seyboth. Onset of deconfinement in nucleus-nucleus collisions: Review for pedestrians and experts. Acta Phys.Polon., B42:307-351, 2011.

[6] S.V. Afanasiev et al. Energy dependence of pion and kaon production in central $\mathrm{Pb}+\mathrm{Pb}$ collisions. Phys.Rev., C66:054902, 2002.

[7] Ekkard Schnedermann, Josef Sollfrank, and Ulrich W. Heinz. Thermal phenomenology of hadrons from 200-A/GeV S+S collisions. Phys.Rev., C48:2462-2475, 1993.

[8] L.D. Landau. On the multiparticle production in high-energy collisions. Izv.Akad.Nauk Ser.Fiz, 17:51-64, 1953.

[9] Edward V. Shuryak. High-Energy Multiple Production of Hadrons and Landau Hydrodynamical Theory. 1974.

[10] J.L. Klay et al. Charged pion production in 2 to 8 agev central au+au collisions. Phys.Rev., C68:054905, 2003.

[11] Ehab Abbas et al. Centrality dependence of the pseudorapidity density distribution for charged particles in Pb-Pb collisions at $\sqrt{s_{\mathrm{NN}}}=2.76$ TeV. Phys.Lett., B726:610-622, 2013.

[12] S.S. Adler et al. Identified charged particle spectra and yields in $\mathrm{Au}+\mathrm{Au}$ collisions at $\mathrm{S}(\mathrm{NN}) * * 1 / 2=$ 200-GeV. Phys.Rev., C69:034909, 2004.

[13] Betty Abelev et al. Pion, Kaon, and Proton Production in Central Pb-Pb Collisions at $\sqrt{s_{N N}}=2.76$ TeV. Phys.Rev.Lett., 109:252301, 2012.

[14] Michael Kliemant, Benjamin Lungwitz, and Marek Gazdzicki. Energy dependence of transverse mass spectra of kaons produced in $\mathrm{p}+\mathrm{p}$ and $\mathrm{p}+$ anti-p interactions: A Compilation. Phys.Rev., C69:044903, 2004.

[15] M. Gazdzicki and D. Roehrich. Pion multiplicity in nuclear collisions. Z.Phys., C65:215-223, 1995.

[16] Marek Gazdzicki and Dieter Rohrich. Strangeness in nuclear collisions. Z.Phys., C71:55-64, 1996.

[17] B.I. Abelev et al. Systematic Measurements of Identified Particle Spectra in $p p, d^{+} \mathrm{Au}$ and $\mathrm{Au}+\mathrm{Au}$ Collisions from STAR. Phys.Rev., C79:034909, 2009.

[18] Betty Bezverkhny Abelev et al. Production of charged pions, kaons and protons at large transverse momenta in pp and $\mathrm{PbPb}$ collisions at $\sqrt{s_{\mathrm{NN}}}=2.76$ TeV. Phys.Lett., B736:196-207, 2014

[19] K. Aamodt et al. Production of pions, kaons and protons in $p p$ collisions at $\sqrt{s}=900 \mathrm{GeV}$ with ALICE at the LHC. Eur.Phys.J., C71:1655, 2011.

[20] I. Arsene et al. Centrality dependent particle production at $\mathrm{y}=0$ and y 1 in $\mathrm{Au}+\mathrm{Au}$ collisions at $\mathrm{s}(\mathrm{NN}) * *(1 / 2)=200-\mathrm{GeV}$. Phys.Rev., C72:014908, 2005.

[21] S.A. Bass, M. Belkacem, M. Bleicher, M. Brandstetter, L. Bravina, et al. Microscopic models for ultrarelativistic heavy ion collisions. Prog.Part.Nucl.Phys., 41:255-369, 1998. 
[22] M. Bleicher, E. Zabrodin, C. Spieles, S.A. Bass, C. Ernst, et al. Relativistic hadron hadron collisions in the ultrarelativistic quantum molecular dynamics model. J.Phys., G25:1859-1896, 1999.

[23] W. Ehehalt and W. Cassing. Relativistic transport approach for nucleus nucleus collisions from SIS to SPS energies. Nucl.Phys., A602:449-486, 1996.

[24] Torbjorn Sjostrand, Stefan Ask, Jesper R. Christiansen, Richard Corke, Nishita Desai, et al. An Introduction to PYTHIA 8.2. 2014.

[25] V. Yu. Vovchenko, D.V. Anchishkin, and M.I. Gorenstein. System-size and energy dependence of particle momentum spectra: The UrQMD analysis of $p+p$ and $\mathrm{Pb}+\mathrm{Pb}$ collisions. Phys.Rev., C90(2):024916, 2014. 